Conclusion The present results suggest that light intensity PA may have an important role in weight control while greater moderateintensity PA may be associated with lower uric acid concentration in individuals with obesity.

\section{SP1-18 ADOLESCENTS' CHRONIC DISEASES AND HEALTH COMPLAINTS: A POPULATION BASED SURVEY IN CAMPINAS, SÃO PAULO, BRAZIL}

doi:10.1136/jech.2011.142976m.95

M Braz, A de Azevedo Barros, ${ }^{*}$ M B de Azevedo Barros. State University of Campinas Medical School, Campinas, São Paulo, Brazil

Introduction The prevalence of diseases has been changing in Brazil in the last decades. The objective of this research was to estimate the prevalence of chronic diseases and health complaints among adolescents according to socioeconomic and demographic variables.

Method A cross-sectional population based survey was carried out in Campinas in the years 2008/09 (ISACamp 2008/09). Data of 929 adolescents from 10 to 19 years were obtained through household interviews. The analysis included prevalence ratio (PR) and 95\% CI. Poisson regression was used for adjusted analysis.

Results $17.54 \%$ (CI 14.34 to 21.28 ) informed having one chronic disease and $1.62 \%$ (0.92 to 2.83) having more than one. Regarding health complaints $38.47 \%$ (31.98 to 45.39 ) referred not having any problem, $33.75 \%$ (29.55 to 38.21 ) informed one problem, $17.31 \%$ (15.46 to 20.58 ) two, $7.23 \%$ (5.27 to 9.83 ) three and $3.23 \%$ (1.88 to 5.56$)$ four or more. Among the chronic diseases, asthma showed the higher prevalence: $7.59 \%$ (6.01 to 9.54). The most prevalent health complaints were: allergy $40.39 \%$ (34.90 to 46.13), frequent headache/migraine $24.83 \%$ (20.35 to 29.92), backache/ column problems $11.84 \%$ (8.97 to 15.48 ) and emotional problems (anxiety and sadness) 10.65\% (7.69 to 14.56). After adjusting for confounding the PR for chronic diseases were: 1.4 (1.06 to 1.82) for age over $15,0.68$ (0.46 to 0.99 ) for those still attending school, and 1.8 (1.06 to 3.18) for having children. The PR for health complaints were: 1.12 (1.01 to 1.24) for girls and 1.34 (1.11 to 1.62) for higher family income.

Conclusion Age from 15 to 19 years, not attending school and having children are associated with chronic diseases, and female adolescent and having a family income of over 4 minimum wages are associated with health complaints.

\section{SP1-19 DIFFUSE REFLECTANCE SPECTRAL IMAGING: A NON- INVASIVE PROMISING TOOL FOR EARLY DIAGNOSIS AND SCREENING OF MALIGNANT CHANGES IN THE ORAL CAVITY}

\section{doi:10.1136/jech.2011.142976m.96}

${ }^{1} \mathrm{M}$ M S Mathunny, ${ }^{* 2} \mathrm{~J}$ L Jayanthi, ${ }^{1} \mathrm{~V} T$ Beena, ${ }^{2} \mathrm{~N}$ Subhash. ${ }^{1}$ Department of Oral and Maxillofacial Pathology, Government Dental College, Trivandrum, Kerala, India; ${ }^{2}$ Biophotonics Laboratory, Centre for Earth Science Studies, Trivandrum, Kerala, India

Background Early detection is vital for improving the survival rate of oral cancer patients.

Materials and Methods Spectral data at 545 and $575 \mathrm{~nm}$ in diffuse reflectance (DR) spectra were imaged from oral lesions of 55 patients and 103 sites of 23 healthy volunteers with an electron multiplying charge coupled device (EMCCD) camera. A computer-generated algorithm based on pixel intensity of DR image ratio (R545/R575) was used for pseudo-colour mapping the entire oral lesion. Tissue biopsies of all patients were taken from selected sites with maximum malignant potential. Spectral data of ratio image were then compared against the histopathology results.

Results The mean age of patients and healthy volunteers were $55.7 \pm 12.0 \mathrm{yrs}$ and $28 \pm 7.0 \mathrm{yrs}$, respectively. Majority of patients were tobacco users (43/55), while 23/55 were users of alcohol. The median pixel intensity value of the ratio-image was significantly lower $(p<0.001)$ in the healthy group $(0.87$, IOR: $0.82-0.94)$ in comparison to pre-malignant (1.35, IOR: 1.13-1.67) and malignant group (2.44, IOR: 1.78-3.80). The area under the ROC curve (AUC) of healthy to pre-malignant, healthy to malignant, pre-malignant to malignant and healthy to pre-malignant/malignant comparisons were 0.94 (95\% CI 0.86 to 1.00), 0.99 (95\% CI 0.99 to 1.00 ), 0.84 (95\% CI 0.73 to 0.95 ) and 0.97 (95\% CI 0.94 to 1.00), respectively. The discriminant function analyses with leave-one-out classification differentiate healthy from pre-malignant/malignant lesions correctly in $84.2 \%$ occasions after cross-validation (Wilks' $\lambda=0.744, \chi^{2}=45.97$ with degree of freedom $=1, \mathrm{p}<0.001$ ).

Conclusion DR spectral imaging efficiently discriminates healthy tissues from oral malignant and pre-malignant lesions.

\section{SP1-20 PREVALENCE OF COGNITIVE IMPAIRMENT AND DEPRESSION AND ASSOCIATED FACTORS IN ELDERS LIVING IN LONG-STAY INSTITUTIONS IN BRAZIL}

doi:10.1136/jech.2011.142976m.97

${ }^{1} \mathrm{~L}$ M Santiago, ${ }^{*}{ }^{1} \mathrm{~L}$ L Luz, ${ }^{1,2} \mathrm{~J}$ F Santos-Silva, ${ }^{1,3} \mathrm{P}$ H Oliveira, ${ }^{1} \mathrm{~L}$ C Alves, ${ }^{1} \mathrm{E}$ E Mattos. ${ }^{1}$ Oswaldo Cruz Foundation, National School of Public Health, Rio de Janeiro, Brazil; ${ }^{2}$ Health Secretariat of Mato Grosso do Sul State, Mato Grosso do Sul/MS, Brazil; ${ }^{3}$ Health Secretariat of Mato Grosso State, Mato Grosso/MT, Brazil

Introduction Cognitive impairment and depression are among the health conditions that affect the ability of the elders to live independently, leading to their placement at long-stay institutions. This phenomenon has been increasing in Brazil. This study analyzes the prevalence of these health conditions and its associated factors in elders who live at these institutions.

Methods This is a cross-sectional study with elderly residents of long-stay institutions in four Brazilian cities. The study's questionnaire included socio-demographic and health-related variables and the assessment of functioning, cognition and mood with scales widely used in elderly populations. The population profile was characterised and the prevalence of cognitive impairment and depression was estimated. Bivariate and multivariate analyses were performed with Poisson regression.

Results These are preliminary results for 340 elders. The mean age was 75.5 years, most were men, with $0-4$ years of schooling and $<5$ years of institutionalisation. The prevalence of cognitive impairment was $65.8 \%$ and of depression $49.1 \%$. Variables associated to cognitive impairment were depression (RP 4.39; 95\% CI 1.11 to 7.27 ) and female gender (RP 2.96; 95\% CI 1.28 to 6.84). The referral of very poor/poor health (RP 2.69; 95\% CI 1.37 to 5.27), hospitalisation in the last year (RP 1.55; 95\% CI 1.04 to 2.33) and presence of cognitive impairment (RP1.42; 95\% CI 1.11 to 1.83 ) were associated to a high probability of depression.

Conclusions The high prevalence of these two conditions in elders living at long-stay institutions in Brazil denotes the importance of health policies aiming to improve the quality of care received by this population group. 